\title{
Mitteilungen
}

\section{Ordentliche Ärztekammer}

Inkraftsetzung der Beschlüsse der Ordentlichen Ärztekammer vom 3. Mai 2007

In der statutarisch festgelegten Frist von 60 Tagen seit Publikation der Beschlüsse in der Schweizerischen Ärztezeitung (SÄZ Nr. 22 vom 30. Mai 2007) ist kein Antrag auf Urabstimmung eingereicht worden. Die Beschlüsse der Ordentlichen Ärztekammer vom 3. Mai 2007 sind am 29. Juli 2007 in Kraft getreten.

\section{Chambre médicale ordinaire}

\section{Entrée en vigueur des décisions}

de la Chambre médicale ordinaire du 3 mai 2007

Aucune demande de votation générale n'ayant été déposée durant le délai statutaire des 60 jours après la publication des décisions de la Chambre médicale ordinaire du 3 mai 2007 dans le Bulletin des médecins suisses (BMS $\mathrm{n}^{\circ} 22$ du 30 mai 2007), elles sont entrées en vigueur le 29 juillet 2007 .

\section{Facharztprüfung}

Facharztprüfung zur Erlangung des Facharzttitels für Oto-Rhino-Laryngologie

Schriftliche Prüfung: Freitag, 23. November 2007, im Hotel Seepark, Thun

Mündliche Prüfung: Donnerstag, 15. November 2007 (Zeit und Örtlichkeiten sind noch nicht bestimmt, je nach Anzahl Kandidaten)

\section{Anmeldefrist: 31. August 2007}

Weitere Informationen finden Sie auf der Website der FMH unter www.fmh.ch/awf $\rightarrow$ Weiterbildung $\rightarrow$ Für Assistenten / Ärzte in Weiterbildung.

\section{Examen de spécialiste}

Examen de spécialiste en vue de l'obtention du titre de spécialiste en oto-rhino-laryngologie

Examen écrit: vendredi 23 novembre 2007 à l'Hôtel Seepark, Thoune

Examen pratique: jeudi 15 novembre 2007 (l'heure et lieux exacts sont encore inconnus et dépendront de nombre de candidats)

\section{Délai d'inscription: le 31 août 2007}

Vous trouverez de plus amples informations sur la site web de la FMH www.fmh.ch/awf $\rightarrow$ Formation postgraduée $\rightarrow$ Pour les assistants / les médecins en formation postgraduée.

\section{TARMED Suisse}

\section{PIK-Entscheide}

Nummer 07012 Vor- und Nachbearbeitungszeit eines Psychotherapeuten

Tarifpositionen: 00.0140, 02.0070, 02.0160, 02.0260, 02.0360

\section{Gültig ab: 25. Juli 2007}

Interpretation: Als Aktenstudium gilt das patientenbezogene Studium von Fremdakten (Lesen und Beurteilung ausführlicher fremder Akten, also nicht das Lesen der selbstverfassten Krankengeschichte des Patienten) inkl. Studiums dort zitierter Literaturstellen. Begründete Ausnahmen für das extensive Aufarbeiten von Eigenakten sind vorbehalten. Dies ist zu dokumentieren und dem Kostenträger auf Anfrage zur Verfügung zu stellen. Eine systematische Verrechnung von Leistungen in Abwesenheit des Patienten zur Einsicht in eigene Dossiers ist nicht zulässig.

\section{Décisions de la CPI}

Numéro 07012 Temps consacré par le psychothérapeute à la préparation du dossier avant et après la consultation

Positions tarif: 00.0140, 02.0070, 02.0160, 02.0260, 02.0360

Valable dès le: 25 juillet 2007
Interprétation: Vaut comme étude du dossier: l'étude de documents tiers relatifs au patient (lecture et évaluation de documents détaillés externes et non pas de ceux rédigés par le médecin et figurant déjà dans le dossier médical du patient), y compris l'étude des articles de référence y cités. Demeure réservée comme exception motivée l'étude extensive de ses propres documents. Ceci doit être documenté et mis à la disposition de l'assureur à sa demande.

La facturation systématique de prestations fournies en l'absence du patient pour l'étude de ses propres documents n'est pas admise.

\section{Schweizerische Gesellschaft für Thorax-, Herz- und Gefässchirurgie Société Suisse de Chirurgie Thoracique et Cardiovasculaire}

Vorstand seit 15. Juni 2007 /

Comité depuis le 15 juin 2007

Präsident / Président

Prof. Markus Furrer, Chur

Vizepräsident / Vice-président

Prof. Peter Stierli, Aarau

Past president / Président sortant Prof. Ludwig K. von Segesser, Lausanne

\section{Sekretär / Secrétaire}

Prof. Thierry Carrel, Bern

\section{Kassier / Trésorier}

Prof. Michele Genoni, Zürich

Beisitzer / Assesseur

Dr Patrick Ruchat, Lausanne; PD Dr. Jürg Schmidli, Bern; Prof. Paul Robert Vogt, Zürich; Prof. Walter Weder, Zürich 
Schweizerische Gesellschaft für Innere Medizin

\section{Preis 2008}

Der Preis 2008 in der Höhe von Fr. 10000.kann für eine bis maximal drei wissenschaftliche Originalarbeiten verliehen werden, welche die folgenden Bedingungen erfüllen:

1. Die Arbeit muss im Jahre 2007 in einer medizinischen Zeitschrift publiziert oder zur Publikation akzeptiert sein. Das Thema der Arbeit muss für die Betreuung von Patienten mit internistischen Krankheiten bedeutend sein oder Grundlagen für diagnostische oder therapeutische Möglichkeiten im Zusammenhang mit internistischen Krankheiten schaffen. Outcome-Studien können eingereicht werden, wenn sie Gebiete der Inneren Medizin berücksichtigen.

2. Schweizer Autoren können auch im Ausland entstandene Arbeiten einreichen.

3. Ausländische Autoren werden berücksichtigt, sofern die eingereichte Arbeit in der Schweiz gemacht wurde.

4. Die Arbeit ist bis zum 31. Dezember 2007 in 5 Exemplaren einzureichen an die Präsidentin der Schweizerischen Gesellschaft für Innere Medizin: Prof. Dr. med. Verena Briner, Departement Medizin, Kantonsspital, 6000 Luzern 16.

\section{Société Suisse de Médecine Interne}

\section{Prix 2008}

Le prix 2008 d'un montant de Fr. 10000.peut être attribué pour un, deux ou au maximum trois travaux scientifiques originaux remplissant les conditions suivantes:

1. Le travail doit avoir été publié dans une revue médicale ou avoir été accepté par celle-ci en 2007, en vue de sa publication. Le thème du travail doit présenter un intérêt particulier pour la prise en charge des patients souffrant de maladies relevant de la médecine interne ou ouvrir la voie à de nouvelles techniques diagnostiques ou thérapeutiques de ce type de maladies. Des études rétrospectives des résultats peuvent être soumises si elles concernent des domaines de la médecine interne.

2. Les auteurs suisses peuvent également présenter des travaux publiés (ou à publier) à l'étranger.

3. Les auteurs étrangers peuvent présenter un travail dans la mesure où il a été réalisé en Suisse.
4. Le travail doit être adressé en 5 exemplaires jusqu'au 31 décembre 2007 à la présidente de la Société Suisse de Médecine Interne: Prof. Dr Verena Briner, Département de médecine, Hôpital cantonal, 6000 Lucerne 16.

\section{Schweizerische Gesellschaft \\ für Pneumologie}

\section{Boehringer-Ingelheim-Pneumo-Preis 2008}

Dank einer grosszügigen Zuwendung der Firma Boehringer Ingelheim kann die Schweizerische Gesellschaft für Pneumologie jährlich einen wissenschaftlichen Preis in der Höhe von Fr. 12 500.- aussetzen. Gemäss den Zweckbestimmungen sollen wissenschaftliche Arbeiten auf dem Gebiet der Erwachsenenund pädiatrischen Pneumologie ausgezeichnet und damit die pneumologische Forschung in der Schweiz gefördert werden.

\section{Anforderungen}

- Der Kandidat / die Kandidatin soll jünger als 40 Jahre sein.

- Die Arbeit soll aus einer/-m schweizerischen Abteilung, Klinik, Institut oder Praxis für Pneumologie stammen.

- Das Manuskript kann in deutscher, französischer oder englischer Sprache abgefasst sein. Es darf am 1. Januar 2007 noch nicht publiziert oder zur Publikation akzeptiert worden sein, kann aber in einer Fachzeitschrift schon eingereicht worden sein.

Der Preis wird anlässlich der Jahrestagung 2008 der Schweizerischen Gesellschaft für Pneumologie in Fribourg verliehen. Die Wahl des Preisträgers / der Preisträgerin erfolgt durch den Vorstand der Schweizerischen Gesellschaft für Pneumologie. Eine Aufteilung des Preises ist nicht vorgesehen.

Kandidaten für die Vergabe des Preises 2008 sind gebeten, ihre wissenschaftliche Arbeit (nur 1 Manuskript pro Kandidat/Kandidatin) mit Lebenslauf und einem kurzen Begleitschreiben (unterschrieben vom Kandidaten selbst) gemäss den obenerwähnten Kriterien bis zum 1. Oktober 2007 an das Zentralsekretariat der Schweizerischen Gesellschaft für Pneumologie, Südbahnhofstrasse 14c, Postfach, 3000 Bern 14 (Tel. 03137820 30, Fax 031 37820 31, E-Mail: e.frey@lung.ch), zu senden. Es ist möglich, sich gleichzeitig für den GlaxoSmithKline- und den Boehringer-IngelheimPneumo-Preis $\mathrm{zu}$ bewerben. In diesem Fall soll der Kandidat/ die Kandidatin im Begleitschreiben vermerken, welche Arbeit für welchen Preis zu den entsprechenden Bedingungen unterbreitet wird.

\section{GlaxoSmithKline-Pneumo-Preis 2008}

Dank einer grosszügigen Zuwendung der Firma GlaxoSmithKline AG kann die Schweizerische Gesellschaft für Pneumologie jährlich einen wissenschaftlichen Preis in der Höhe von Fr. 12 500.- aussetzen. Gemäss den Zweckbestimmungen sollen wissenschaftliche Arbeiten auf dem Gebiet der Erwachsenenund pädiatrischen Pneumologie ausgezeichnet und damit die pneumologische Forschung in der Schweiz gefördert werden.

\section{Anforderungen}

- Der Kandidat/die Kandidatin soll jünger als 40 Jahre sein.

- Die Arbeit soll aus einer/-m schweizerischen Abteilung, Klinik, Institut oder Praxis für Pneumologie stammen.

- Das Manuskript kann in deutscher, französischer oder englischer Sprache abgefasst sein. Es muss von einer peer-reviewed Zeitschrift entweder zur Publikation akzeptiert oder seit dem 1. Januar 2007 publiziert worden sein.

Der Preis wird anlässlich der Jahrestagung 2008 der Schweizerischen Gesellschaft für Pneumologie in Fribourg verliehen. Die Wahl des Preisträgers / der Preisträgerin erfolgt durch den Vorstand der Schweizerischen Gesellschaft für Pneumologie. Eine Aufteilung des Preises ist nicht vorgesehen.

Kandidaten für die Vergabe des Preises 2008 sind gebeten, ihre wissenschaftliche Arbeit (nur 1 Manuskript pro Kandidat/Kandidatin) zusammen mit einem Bestätigungsschreiben der Zeitschrift, dass die Arbeit demnächst publiziert wird, dem Lebenslauf und einem kurzen Begleitschreiben (unterschrieben vom Kandidaten selbst) gemäss den obenerwähnten Kriterien bis zum 1. Oktober 2007 an das Zentralsekretariat der Schweizerischen Gesellschaft für Pneumologie, Südbahnhofstr, 14c, Postfach, 3000 Bern 14 (Tel. 03137820 30, Fax 03137820 31, E-Mail: e.frey@lung.ch), zu senden.

Es ist möglich, sich gleichzeitig für den Boehringer-Ingelheim- und den GlaxoSmithKline-Pneumo-Preis zu bewerben. In diesem Fall soll der Kandidat / die Kandidatin im Begleitschreiben vermerken, welche Arbeit für welchen Preis zu den entsprechenden Bedingungen unterbreitet wird. 


\section{Société Suisse de Pneumologie}

\section{Prix Pneumo Boehringer Ingelheim 2008}

Grâce à la générosité de la maison Boehringer Ingelheim, la Société Suisse de Pneumologie a pu créer un prix scientifique d'un montant annuel de Fr. 12 500.-. Son but est d'encourager et de distinguer des travaux scientifiques dans le domaine de la pneumologie adulte et pédiatrique en Suisse.

\section{Exigences}

- Le candidat / la candidate doit avoir moins de 40 ans.

- Le travail doit provenir d'un(e) service, clinique, institut ou cabinet médical suisse de pneumologie.

- Le manuscrit peut être rédigé en allemand, français ou anglais. Il ne doit pas encore avoir été publié ou accepté pour publica-

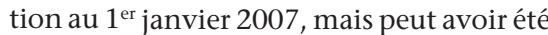
déjà adressé à une revue spécialisée.

Le prix sera remis au cours de l'Assemblée annuelle de la Société Suisse de Pneumologie à Fribourg en 2008. C'est le Comité de la Société Suisse de Pneumologie qui choisit le lauréat / la lauréate. Un partage du prix n'est pas prévu. Les candidats pour l'attribution du prix 2008 sont priés d'adresser leur travail scientifique (1 seul manuscrit par candidat/candidate), accompagné de leur curriculum vitae et d'une courte lettre d'accompagnement, qu'ils auront signé eux-mêmes, à l'adresse suivante jusqu'au $1^{\text {er }}$ octobre 2007: Secrétariat central de la Société Suisse de Pneumologie, Südbahnhofstrasse 14c, case postale, 3000 Berne 14 (tél. 03137820 30, fax 03137820 31, e-mail: e.frey@lung.ch).

Il est possible de se porter simultanément candidat pour le Prix de Pneumologie GlaxoSmithKline et pour le Prix de Pneumologie Boehringer Ingelheim. Dans ce cas, le candidat/la candidate doit mentionner dans sa lettre d'accompagnement quel travail est soumis pour quel prix selon les conditions respectives.

\section{Prix Pneumo GlaxoSmithKline 2008}

Grâce à la générosité de la maison GlaxoSmithKline SA, la Société Suisse de Pneumologie a pu créer un prix scientifique d'un montant annuel de Fr. 12500.-. Son but est d'encourager et de distinguer des travaux scientifiques dans le domaine de la pneumologie adulte et pédiatrique en Suisse.

\section{Exigences}

- Le candidat / la candidate doit avoir moins de 40 ans.

- Le travail doit provenir d'un(e) service, clinique, institut ou cabinet médical suisse de pneumologie.

- Le manuscrit peut être rédigé en allemand, français ou anglais. Il doit être soit accepté pour publication soit avoir été publié dans une revue à politique éditoriale dès le $1^{\text {er }}$ janvier 2007.

Le prix sera remis au cours de l'Assemblée annuelle de la Société Suisse de Pneumologie à Fribourg en 2008. C'est le Comité de la Société Suisse de Pneumologie qui choisit le lauréat / la lauréate. Un partage du prix n'est pas prévu. Les candidats pour l'attribution du prix 2008 sont priés d'adresser leur travail scientifique (1 seul manuscrit par candidat/candidate), la lettre d'acceptation de la revue si le travail est «in press» accompagné de leur curriculum vitae et d'une courte lettre d'accompagnement, qu'ils auront signé eux-mêmes, à l'adresse suivante jusqu'au $1^{\text {er }}$ octobre 2007: Secrétariat central de la Société Suisse de Pneumologie, Südbahnhofstrasse $14 \mathrm{c}$, case postale, 3000 Berne 14 (tél. 03137820 30, fax 03137820 31, e-mail: e.frey@lung.ch).

Il est possible de se porter simultanément candidat pour le Prix de Pneumologie Boehringer Ingelheim et pour le Prix de Pneumologie GlaxoSmithKline. Dans ce cas, le candidat / la candidate doit mentionner dans sa lettre d'accompagnement quel travail est soumis pour quel prix selon les conditions respectives.

\section{Chargenrückruf}

Retin-A ${ }^{\circledast}$ Crème $0,025 \%$,

\section{Zulassungsvermerk 3796103}

Wir möchten Sie darüber informieren, dass die Janssen-Cilag AG folgende Chargen von Retin- $A^{\circledR}$ Crème 0,025\% (EAN 7680379610272 und Pharmacode 1373602) auf der Stufe Grossisten zurückruft: 7BS3K00, 6LSOV00, 6JS1F00, 6FS3B00 und 6DS3P00.
Es handelt sich dabei um die seit April 2006 hergestellten Chargen von Retin- $A^{\circledR}$ Crème 0,025\%. Die anderen Formulierungen (Retin$\mathrm{A}^{\circledR}$ Crème $0,05 \%$ und Retin- $\mathrm{A}^{\circledR}$ Gel $0,025 \%$ ) und die vor April 2006 hergestellten Chargen von Retin- $A^{\circledR}$ Crème $0,025 \%$ sind von diesem Rückruf nicht betroffen.

Grund für den Rückruf: Routinestabilitätsprüfungen haben ergeben, dass der Wirkstoffgehalt der Retin- $A^{\circledR}$ Crème $0,025 \%$ vor Erreichen des Verfalldatums unter die Spezifikationsgrenze fallen kann. Die Firma untersucht zur Zeit die Ursache für die Stabilitätsprobleme. Ein Sicherheitsrisiko für den Patienten besteht nicht.

Retin- $A^{\circledR}$ Crème $0,025 \%$ wird von JanssenCilag AG bis zur genauen Klärung der Qualitätsprobleme nicht weiter ausgeliefert. Retin- ${ }^{\circledR}$ Crème $0,05 \%$ und Retin- $\mathrm{A}^{\circledR}$ Gel $0,025 \%$ stehen weiterhin zur Verfügung.

Dieser Rückruf erfolgt im Einvernehmen mit Swissmedic.

Janssen-Cilag AG, Baar

\section{Retrait de lot}

Retin- $\mathrm{A}^{\circledR}$ Crème $0,025 \%$, numéro d'autorisation 3796103

Nous vous informons ci-après que JanssenCilag AG demande le retour au niveau des grossistes des lots suivants de Retin- ${ }^{\circledR}$ Crème 0,025\% (EAN 7680379610272 et Pharmacode 1373602): 7BS3K00, 6LSOV00, 6JS1F00, $6 \mathrm{FS} 3 \mathrm{~B} 00$ et 6DS3P00.

Il s'agit de lots de Retin- $A^{\circledR}$ Crème $0,025 \%$ fabriqués depuis avril 2006. Les autres formulations de ce produit (Retin- $\mathrm{A}^{\circledast}$ Crème $0,05 \%$ et Retin- $\mathrm{A}^{\circledR}$ Gel $0,025 \%$ ) et les lots de Retin$\mathrm{A}^{\circledR}$ Crème $0,025 \%$ fabriqués avant avril 2006 ne sont pas concernés par ce rappel.

Raison du rappel: Des tests de stabilité routiniers ont montré que la teneur en principe actif de Retin- $\mathrm{A}^{\circledR}$ Crème $0,025 \%$ peut tomber au-dessous des limites des spécifications avant la date de péremption. La maison JanssenCilag AG recherche en ce moment la cause de ce problème de stabilité. Le patient ne court aucun risque.

Retin- $\mathrm{A}^{\circledast}$ Crème $0,025 \%$ ne sera plus livrée par Janssen-Cilag AG avant que ce problème de qualité n'ait été élucidé. Retin- ${ }^{\circledR}$ Crème $0,05 \%$ et Retin- $A^{\circledR}$ Gel 0,025\% continuent d'être à votre disposition.

Ce rappel est effectué en accord avec Swissmedic.

Janssen-Cilag AG, Baar 